\title{
Phonon-mediated negative differential conductance in molecular quantum dots
}

\author{
Alex Zazunov \\ Centre de Physique Théorique, Case 907 Luminy, 13288 Marseille cedex 9, France and \\ Laboratoire d'Etudes des Propriétes Electroniques des Solides, CNRS, BP 166, 38042 Grenoble, France \\ Denis Feinberg \\ Laboratoire d'Etudes des Propriétes Electroniques des Solides, CNRS, \\ associated with Université Joseph Fourier, BP166, 38042 Grenoble, France \\ Thierry Martin \\ Centre de Physique Théorique and Université de la Méditerannée, \\ Case 907 Luminy, 13288 Marseille cedex 9, France
}

(Dated: November 16, 2018)

\begin{abstract}
Transport through a single molecular conductor is considered, showing negative differential conductance behavior associated with phonon-mediated electron tunneling processes. This theoretical work is motivated by a recent experiment by Leroy et al. using a carbon nanotube contacted by an STM tip [Nature 432, 371 (2004)], where negative differential conductance of the breathing mode phonon side peaks could be observed. A peculiarity of this system is that the tunneling couplings which inject electrons and those which collect them on the substrate are highly asymmetrical. A quantum dot model is used, coupling a single electronic level to a local phonon, forming polaron levels. A "half-shuttle" mechanism is also introduced. A quantum kinetic formulation allows to derive rate equations. Assuming asymmetric tunneling rates, and in the absence of the half-shuttle coupling, negative differential conductance is obtained for a wide range of parameters. A detailed explanation of this phenomenon is provided, showing that NDC is maximal for intermediate electronphonon coupling. In addition, in absence of a gate, the "floating" level results in two distinct lengths for the current plateaus, related to the capacitive couplings at the two junctions. It is shown that the "half-shuttle" mechanism tends to reinforce the negative differential regions, but it cannot trigger this behavior on its own.
\end{abstract}

\section{INTRODUCTION}

The prospect of using molecules as the fundamental building blocks of future nanoelectronics devices is rather innovating and exciting from the point of view of potential applications. On the fundamental side, the field of molecular electronics also opens new directions for research because of the prominent role of phonon (vibron) excitations in electronic transport. The nano-objects which are connected to metallic leads may consists of individual molecules, self-assembled monolayers, or conjugated systems such as polymers and carbon nanotubes. The interplay of electron transport and molecular vibrations has triggered much interest, and unambiguous signatures of phonons have been detected in several experiments 1 2.3.4.5.6.7.8.

A recent work ${ }^{7.8}$ considered electron injection from a scanning tunneling microscope (STM) tip into a carbon nanotube. A single-wall carbon nanotube (CNT) is freely suspended over a trench. The STM-tip is located near the center of the suspended part of the CNT. A DC-bias voltage $V$ is applied between the substrate and the STM, and the current flowing through the STM-tip - CNT - substrate structure is measured at a given tunneling distance, controlled by the setpoint current. The motivation for this special geometry is to allow for free internal vibrations to occur in the suspended portion of the nanotube, in particular the so-called radial breathing modes (RBM) $\frac{9}{\underline{n}}$. Contrary to acoustic modes in such system, this mode has a rather high oscillation frequency, and the authors observed that conductance peaks in the current-voltage characteristics of the nanotube were surrounded by phonon side peaks, due to emission or absorption of RBM phonons. Indeed, CNT display a sizeable coupling of electrons to RBM modes 10 . The contacts to the nanotube being rather resistive, transport in this system is dominated by the Coulomb blockade regime, and phonon side peaks were observed around each Coulomb blockade peak in differential conductance plots.

Interestingly, the authors mention frequent detection of negative differential conductance (NDC) regions. Striking NDC features also appear in a very recent work by Sapmaz et $a l^{6}$, , in a transport measurement of a suspended CNT, where current flows through the CNT, between two contacts at the substrate. In this work, phonon side peaks are attributed to longitudinal stretching modes, and the steps in the $I(V)$ characteristics are followed by spikes, thus displaying NDC features. The purpose of the present theoretical work is to show that such NDC features can be described quite simply using a generic model which consists of a quantum dot with a single orbital level, coupled to on-site single phonon mode, and connected to leads by tunnel junctions. Due to the weak tunnel couplings, the physics of NDC appear to be a consequence of the transport through small polaron states on the molecule. Notice 
that polaron formation in CNT is suggested by several works ${ }^{10}$. Similar models have previously been considered in the literature11.12.13.14.15.16.17. Here we consider a molecular system - or a nanotube setup - whose tunneling matrix elements from the molecule to the leads are asymmetric, which is the case in Ref. 7, and possibly also in Ref. 6. We propose that given this asymmetry, NDC can be obtained for a wide range of parameters and it can even be quantified by analytical means for strong asymmetry. Furthermore, we argue that our approach for the description of NDC is by no means confined to the experimental geometry of Ref. 7, which can be considered as an "experimental paradigm" for phonon modulated molecular transport. The present approach should apply to any molecular transport in the Coulomb blockade regime where an optical-like phonon mode dominates. Notice that NDC was found in Ref 11 within a symmetric model, using an ansatz for the Franck-Condon factors coupling the leads to the polaron levels. Catastrophic NDC was also proposed to occur in Ref. 13, but due to an additional mechanism. In the framework of a single site model, NDC was also found in an adiabatic treatment of the polaron problem ${ }^{21}$. It has been proposed to occur in the case of two competing molecular states $\frac{18}{2}$ or within a two-site mode 19 . Besides electron-phonon coupling, other physical mechanisms can lead to NDC20.

In molecular electronics, one is tempted to use existing theoretical tools developed in the context of mesoscopic physics because the size of the devices allows a coherent description of the transport inside the molecule. When the molecule is connected to good contacts, and when the role of Coulomb interaction and electron-phonon interactions is reduced, the Landauer formulation of transport combined with a Green's function calculation of the transmission coefficient serves as a good starting point for computing transport 22 , but it then neglects the dynamical degrees of freedom of the molecule. Phonons can be included in these approaches, analytically ${ }^{23}$, or using numerical calculations ${ }^{24.25}$ based on the work of Ref. 26. Alternatively, the Landauer approach can be generalized to a situation where interactions are restricted to a finite region ${ }^{12.15 .27}$ using non-equilibrium Green's functions.

On the other hand, molecular contacts or the contacts to a nanotube are often of poor quality. It is then reasonable to think of the molecule as a quantum dot, which is subject to the Coulomb blockade. This point of view was adopted by several authors $11,12,13,15,16,28$, and is confirmed by experiments. One of the advantages of modecular electronics comes from the size of the individual nano-object which is connected to the electrodes. Even at relatively large temperatures (a few Kelvins), the dynamics within the molecule/nanotube is fully phase coherent. On the other hand, the tunneling rates to the reservoirs are typically small compared to temperature. One can then derive rate equations for the electron population of the dot. Here our goal is to use the simplest model which can account for the physics of the experiment of Ref. 7. We therefore derive a kinetic equation approach which can account for the observed NDC effect, treating the electron-phonon coupling non-perturbatively while still allowing for an intuitive understanding of the physics at hand. Furthermore, consequences can be derived on the electron transport as well as on the non-equilibrium phonon population. In the present work the local mode is not coupled to any environmental degrees of freedom, like phonon modes or electron-hole excitations in the substrate. Although such coupling is probably relevant in some experiments, the very high phonon quality factors $(Q>20000)$ obtained in other works ${ }^{7}$ justify to neglect it as a first step.

Experimentally, the addition of voltage gates on a molecular transport setup is still challenging but it is possible 8.29 to approach three metallic probes in a nanometer scale region. Yet, there is also a motivation to study a setup where the molecular levels are "floating" instead of being fixed by a gate. Besides the strong asymmetry of the tunneling rates, a specific feature of our work is that NDC can occur in such absence of gate voltage, and that the ratio of the right/left capacitance plays an important role, as it dictates the location of the molecular levels.

The structure of the paper is as follows. The model is introduced in Sec. II, and the derivation of the rate equations is provided in Sec. III. The general considerations about current transport and phonon occupation numbers are discussed in Sec. IV. Numerical results are presented in Sec. V, illustrating the role of the asymmetry of the capacitances, of the shift of the dot level, and of the so-called half-shuttle mechanism on the NDC. We conclude in Sec. VI.

\section{THEORETICAL MODEL}

Although the potential relevance of our approach applies to the carbon nanotube experiments of Refs. 7,8, we refer to the central region between the leads as the quantum dot. This quantum dot is weakly coupled to two metallic electrodes by tunnel junctions. In the STM geometry of Refs. 7,8, the electrodes represent the STM-tip and the substrate, to which we refer as the 'left' $(l)$ and 'right' $(r)$ electrodes, respectively. Each tunnel junction $(j=l, r)$ is characterized by a resistance $\left(R_{j}\right)$ and a capacitance $\left(C_{j}\right)$. While $R_{r}$ and $C_{r}$ are constant for a given contacted nanotube sample, $R_{l}$ and $C_{l}$ are functions of the tip-tube separation. The capacitances $C_{l}$ and $C_{r}$ are sample-dependent ${ }^{30}$. For generality, one can add a gate voltage $V_{g}$ and a gate capacitance $C_{g}$.

We focus on the strong Coulomb blockade regime, assuming that the number of electrons which can be added to the dot is restricted to one. The physical features revealed in the corresponding bias window can be easily extended to a 


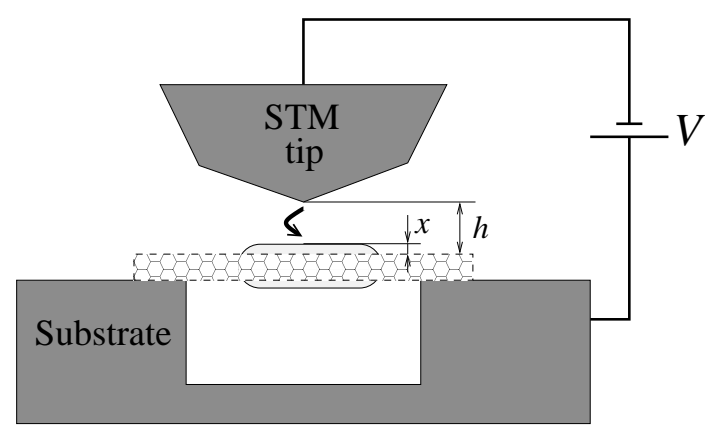

FIG. 1: Schematic drawing of a carbon nanotube suspended over a trench. A bias voltage is applied between the STM tip (source) and substrate (drain). The inflated portion in the suspended portion of the nanotube illustrates the radial breathing mode. $h$ is the tip-nanotube separation at rest.

full span of many Coulomb blockade peaks, as well a several orbital levels as in Refs. 7,8. The extra-charge electron state is locally coupled to a phonon mode: in Refs. 7,8 this mode is identified with the radial breathing mode (RBM) of the carbon nanotube ${ }^{9.10}$ and, in Ref. 6, with the longitudinal stretching mode. In view of the phonon-assisted tunneling processes, the above-mentioned Coulomb blockade regime implies that the charging energy of the dot is assumed to be infinitely large compared to the relevant energy scale determined (at low temperature) by the phonon energy $\Omega$ and the bias voltage. In the absence of phonons, electron transport would occur only through a single electron state on the dot.

In the Coulomb blockade regime, far from the Kondo regime, spin degrees of freedom are neglected. The Hamiltonian of the system is written as follows:

$$
\mathcal{H}=\mathcal{H}_{0}+\mathcal{H}_{\text {leads }}+\mathcal{V}
$$

where

$$
\begin{gathered}
\mathcal{H}_{0}=(\epsilon-g x) d^{\dagger} d+\Omega b^{\dagger} b, \quad x=b+b^{\dagger} \\
\mathcal{H}_{\text {leads }}=\sum_{j k} \xi_{j k} c_{j k}^{\dagger} c_{j k} \\
\mathcal{V}=\sum_{j k} \mathcal{T}_{j}(x) c_{j k}^{\dagger} d+\text { h.c. }
\end{gathered}
$$

Here the operator $d\left(d^{\dagger}\right)$ annihilates (creates) an electron on a single dot level of energy $\epsilon$; similarly, $c_{j k}\left(c_{j k}^{\dagger}\right)$ annihilates (creates) an electron with momentum $k$ and energy $\xi_{j k}$ in the $j$ th lead. The RBM is linearly coupled (with the coupling energy $g$ ) to the electric charge on the dot; the RBM excitations are annihilated (created) by $b\left(b^{\dagger}\right)$. In Eq. (4), $\mathcal{T}_{j}$ is the energy associated with the tunneling coupling to the dot; for simplicity, $\mathcal{T}_{j}$ is assumed to be energy-independent (constant density of states in the metallic leads), but we take into account the dependence of the tip-tube tunneling matrix amplitude on the boson coordinate $x$ : due to the "breathing" motion of the tube, the tip-tube tunneling distance deviates from its equilibrium value. Explicitly, we assume an exponential $x$-dependence:

$$
\mathcal{T}_{l}(x)=\mathcal{T}_{l 0} e^{-s x},
$$

where $s$ is determined by the ratio of the amplitude of the zero-point RBM oscillations to the electronic tunneling length $(\approx 0.5 \AA)$ which characterizes the tunnel barrier between the STM tip and the nanotube. Such position dependent amplitudes have been introduced in the context of nano-mechanical electronic devices ${ }^{31.32 .33 .34}$, where one refers to the "shuttle" mechanism as the central region oscillates between the two electrodes. On the other hand, in our situation the tube-substrate tunneling matrix amplitude is $x$-independent: $\mathcal{T}_{r}(x) \equiv \mathcal{T}_{r 0}$. For $s \neq 0$, we refer here to this position dependent tunneling Hamiltonian as the "half-shuttle" mechanism, because only one of the tunneling amplitude (left) is modified by the position.

In the model Hamiltonian (11), formally describing the phonon-assisted tunneling of otherwise noninteracting electrons, the charging effects are taken into account via the bias-voltage dependence of the position of the dot level $(\epsilon)$ 
with respect to the chemical potentials of the leads, $\mu_{l, r}$. A gate voltage $V_{g}$ and gate capacitance $C_{g}$ can be included. In our analysis, $\epsilon$ includes the change in the charging energy of the dot when one extra electron is added to the dot, and which is obtained from the electrostatic energy consideration:

$$
\epsilon-\epsilon_{0}=2 E_{C}\left(n_{x}+1 / 2\right)+e \phi
$$

where $\epsilon_{0}$ is the "bare" energy level of the electron level in the dot, $E_{C}=e^{2} / 2 C$ is the charging energy and $e n_{x}$ is a background (fractional) charge of the dot. Furthermore, the potential of the dot, $\phi$, is given by

$$
\begin{gathered}
\phi=\sum_{j=l, r} c_{j} \mu_{j} / e+c_{g} V_{g}, \\
c_{j}=C_{j} / C, \quad c_{g}=C_{g} / C, \quad C=C_{l}+C_{r}+C_{g} .
\end{gathered}
$$

In typical experiments $C_{g} \ll C_{j}$. It is worth noticing that in the "floating-level" geometry which is considered in part of this work (e. g. without the gate electrode, $C_{g}=0$ ), the fractional charge is not fixed but it can instead be affected by changes in the capacitances of the junctions 35 (that is, $n_{x}$ may depend on the tip-tube separation).

Assuming weak coupling to the leads, it is convenient to eliminate the electron-phonon coupling in $\mathcal{H}_{0}$ by the unitary transformation $\tilde{\mathcal{H}}=\Lambda^{\dagger} \mathcal{H} \Lambda$, with

$$
\Lambda=e^{-i \alpha p d^{\dagger} d}, \quad p=-i\left(b-b^{\dagger}\right), \quad \alpha=g / \Omega,
$$

In the rotated basis, the electron state in the dot becomes "dressed" with phonons, forming a small polaron. This results in the "polaron shift" of the dot level energy, $\tilde{\epsilon}=\epsilon-g^{2} / \Omega$, and a renormalization of the dot-lead tunneling coupling. In the polaron representation, the Hamiltonian reads:

$$
\begin{aligned}
\tilde{\mathcal{H}} & =\tilde{\mathcal{H}}_{0}+\tilde{\mathcal{V}}+\mathcal{H}_{\text {leads }} \\
\tilde{\mathcal{H}}_{0} & =\epsilon d^{\dagger} d+\Omega b^{\dagger} b \\
\tilde{\mathcal{V}} & =\sum_{j k} \mathcal{T}_{j}(x) e^{-i \alpha p} c_{j k}^{\dagger} d+\text { h.c. }
\end{aligned}
$$

In Eq. (12), we have used the fact that

$$
\Lambda^{\dagger} \mathcal{T}_{l}(x) d \Lambda=\mathcal{T}_{l}\left(x+2 \alpha d^{\dagger} d\right) d e^{-i \alpha p}=\mathcal{T}_{l}(x) e^{-i \alpha p} d
$$

\section{RATE EQUATIONS}

Based on the assumption that the leads are in thermal equilibrium at given chemical potentials $\left(\mu_{l}\right.$ and $\left.\mu_{r}\right)$, independently of the state of the dot, one can derive a kinetic equation for the reduced density matrix by tracing out the electrode degrees of freedom from the total density matrix. Such equations have been used by several authors, 11.13 .16 .32 but for sake of completeness we provide here a full derivation.

In the polaron representation, the reduced density matrix of the phonon-coupled dot is given by

$$
R(t)=e^{-i t \tilde{\mathcal{H}}_{0}} R_{V}(t) e^{i t \tilde{\mathcal{H}}_{0}}, \quad R_{V}(t)=\operatorname{Tr}_{l e a d s} \tilde{\rho}_{V}(t)
$$

where $\tilde{\rho}_{V}(t)$ is the total density matrix in the interaction picture (with respect to the tunneling coupling to the leads), which obeys the operator equation of motion:

$$
\begin{array}{r}
i \partial_{t} \tilde{\rho}_{V}(t)=\left[\tilde{\mathcal{V}}(t), \tilde{\rho}_{V}(t)\right] \\
\tilde{\mathcal{V}}(t)=e^{i t\left(\tilde{\mathcal{H}}_{0}+\mathcal{H}_{\text {leads }}\right)} \tilde{\mathcal{V}} e^{-i t\left(\tilde{\mathcal{H}}_{0}+\mathcal{H}_{\text {leads }}\right)} .
\end{array}
$$

Using the integral form of Eq. (15),

$$
\tilde{\rho}_{V}(t)=-i \int_{0}^{t} d \tau\left[\tilde{\mathcal{V}}(\tau), \tilde{\rho}_{V}(\tau)\right]+\tilde{\rho}_{V}(0)
$$


substituting Eq. (17) into Eq. (15), and taking the trace over the electronic degrees of freedom of the leads with the above mentioned assumption of $\tilde{\rho}_{V}(t)=\rho_{\text {leads }}^{e q} \otimes R_{V}(t)$, where $\rho_{\text {leads }}^{e q}$ is the equilibrium density matrix of the leads, we arrive at the integro-differential equation of motion for the reduced density matrix in the polaron representation:

$$
\begin{array}{r}
\partial_{t} R_{V}(t)=-\sum_{j=l, r} 2 \pi \nu_{j} \int_{0}^{t} d \tau e^{i \tilde{\epsilon} \tau}\left[F_{j}^{>}(\tau)\left\{\tilde{\mathcal{T}}_{j}^{\dagger}(t) \tilde{\mathcal{T}}_{j}(t-\tau) d^{\dagger} d R_{V}(t-\tau)-\tilde{\mathcal{T}}_{j}(t-\tau) d R_{V}(t-\tau) d^{\dagger} \tilde{\mathcal{T}}_{j}^{\dagger}(t)\right\}\right. \\
\left.+F_{j}^{<}(\tau)\left\{R_{V}(t-\tau) d d^{\dagger} \tilde{\mathcal{T}}_{j}(t-\tau) \tilde{\mathcal{T}}_{j}^{\dagger}(t)-\tilde{\mathcal{T}}_{j}^{\dagger}(t) d^{\dagger} R_{V}(t-\tau) d \tilde{\mathcal{T}}_{j}(t-\tau)\right\}\right]+h . c .
\end{array}
$$

where $\nu_{j}$ is the density of states in the $j$ th electrode,

$$
\tilde{\mathcal{T}}_{j}(\tau)=\mathcal{T}_{j}\left[(x(\tau)] e^{-i \alpha p(\tau)}\right.
$$

with

$$
\begin{aligned}
& x(\tau)=b e^{-i \Omega \tau}+h . c . \\
& p(\tau)=-i b e^{-i \Omega \tau}+h . c .
\end{aligned}
$$

and

$$
F_{j}^{>,<}(\tau)=\frac{1}{2}\left[\delta(\tau) \mp \text { P.v. } \frac{i e^{-i \mu_{j} \tau}}{\beta \sinh (\pi \tau / \beta)}\right]
$$

(where "P.v." stands for the principal value). From the above expressions for the kernels $F_{j}^{>,<}(\tau)$, it follows that the relevant retardation time is of the order of the inverse temperature, $\beta$. Assuming temperature to be high compared to the tunneling rates, $\beta^{-1} \gg 2 \pi \nu_{j} \mathcal{T}_{j}^{2}$, and considering the long-time behavior of the reduced density matrix, $t \gg \beta$, we construct an asymptotic solution of Eq. (18) by using an improved perturbation expansion ${ }^{36}$ :

$$
R_{V}(t)=\bar{R}(\lambda t)+\lambda R^{(1)}(t)+o(\lambda) .
$$

In Eq. (23), $\lambda$ is a formal perturbation parameter that reflects a weakness of tunnel coupling and must be formally attributed to the right-hand side of Eq. (18):

$$
\lambda \partial_{z} \bar{R}(z)+\lambda \partial_{t} R^{(1)}(t)+o(\lambda)=-\lambda \sum_{j=l, r} 2 \pi \nu_{j} \int_{0}^{t} d \tau e^{i \tilde{\epsilon} \tau}\left[F _ { j } ^ { > } ( \tau ) \left\{\tilde{\mathcal{T}}_{j}^{\dagger}(t) \tilde{\mathcal{T}}_{j}(t-\tau) d^{\dagger} d \bar{R}(z-\lambda \tau)+\ldots\right.\right.
$$

with $z=\lambda t$. In the first-order equation in $\lambda$, retardation effects are neglected (the Markovian approximation), and in the above equation, we can replace $\int_{0}^{t} d \tau \ldots \rightarrow \int_{0}^{+\infty} d \tau \ldots$. By constraction, $\bar{R}$ is, on the time scale $\beta$, a slowly evolving part of the reduced density matrix, while the rapidly oscillating (with frequencies determined by a multiple integer of $\Omega$ ) part of the right-hand side of Eq. (24) is absorbed into $R^{(1)}$. In what follows, we assume that the phonon frequency is high compared to the tunneling rates, $\Omega \gg 2 \pi \nu_{j} \mathcal{T}_{j}^{2}$. This "anti-adiabatic" condition allows us to neglect $R^{(1)}$ as being negligibly small compared to $\bar{R}$. Under the above assumptions, Eq. (24) reduces to a differential operator equation for $\bar{R}(t)$.

Although $\bar{R}$ is diagonal in the on-dot electron number (no coherence between subsequent tunneling events at long times),

$$
\bar{R}(t)=\left(1-d^{\dagger} d\right) R^{0}(t)+d^{\dagger} d R^{1}(t)
$$

yet it is non-diagonal in the phonon number due to the presence of the displacement operators $\left(e^{ \pm i \alpha p}\right)$ in the righthand side of Eq. (24). However, equations for the diagonal and off-diagonal parts of $\bar{R}(t)$ are decoupled from each other. As a result, in steady state $(t \rightarrow+\infty)$, which we are interested in, $\bar{R}$ becomes diagonal in both the electron and phonon numbers.

Introducing the electron-phonon joint probabilities

$$
P_{n}^{i}(t)=\left\langle n\left|R^{i}(t)\right| n\right\rangle
$$

for $i=\{0,1\}$ additional electrons and $n=\{0,1,2, \ldots\}$ boson excitations on the dot, we obtain the system of rate equations for the joint probabilities, which in steady case can be written in the following form:

$$
\begin{aligned}
& \partial_{t} P_{n}^{0}=0=-\Gamma_{n}^{<} P_{n}^{0}+\sum_{m} L_{n m}^{>} P_{m}^{1}, \\
& \partial_{t} P_{n}^{1}=0=-\Gamma_{n}^{>} P_{n}^{1}+\sum_{m} L_{n m}^{<} P_{m}^{0},
\end{aligned}
$$


with the normalization condition, $\sum_{n}\left(R_{n}^{0}+R_{n}^{1}\right)=1$. The charge/phonon transition rates are given by

$$
\begin{gathered}
\Gamma_{n}^{<}=\sum_{m} L_{m n}^{<}, \quad \Gamma_{n}^{>}=\sum_{m} L_{m n}^{>} \\
L_{n m}^{<}=\sum_{j=l, r} \Gamma_{j} \gamma_{j, m n}^{2} f_{j}\left(\tilde{\epsilon}-\Omega_{m n}\right), \quad L_{n m}^{>}=\sum_{j=l, r} \Gamma_{j} \gamma_{j, n m}^{2}\left(1-f_{j}\left(\tilde{\epsilon}-\Omega_{n m}\right)\right) .
\end{gathered}
$$

In the above equations, $f_{j}(\omega)=\left[e^{\beta\left(\omega-\mu_{j}\right)}+1\right]^{-1}$ is the Fermi distribution function of the $j$ th electrode, $\Omega_{n m}=$ $(n-m) \Omega$,

$$
\begin{gathered}
\Gamma_{j}=2 \pi \nu_{j} \mathcal{T}_{j 0}^{2}, \\
\gamma_{l, n m}=\left\langle n\left|e^{-s x} e^{-i \alpha p}\right| m\right\rangle, \quad \gamma_{r, n m}=\left\langle n\left|e^{-i \alpha p}\right| m\right\rangle .
\end{gathered}
$$

Explicitly, we have for the oscillator matrix elements:

$$
\left\langle n\left|e^{-s x} e^{-i \alpha p}\right| m\right\rangle=e^{-s \alpha+\frac{1}{2}\left(s^{2}-\alpha^{2}\right)} \sum_{q=0}^{\operatorname{Min}(n, m)} \frac{(-1)^{m-q} \sqrt{n ! m !}}{(n-q) !(m-q) ! q !}(\alpha-s)^{n-q}(\alpha+s)^{m-q} .
$$

From the solution of Eq. (27) we can calculate the DC current and expectation values of the phonon observables. It should be noticed that in the original (non-polaron) representation, the resulting phonon density matrix is given by

$$
R_{p h}=\sum_{n}\left(P_{n}^{0}|n\rangle\left\langle n\left|+P_{n}^{1} e^{-i \alpha p}\right| n\right\rangle\langle n| e^{i \alpha p}\right) .
$$

In general, $R_{p h}$ has contributions from both charge states $i=0,1$ and is non-diagonal in vibrational space (two shifted oscillators).

In order to express the current in terms of the joint probabilities $P_{n}^{i}$, we have to average the current operator taken in the polaron representation,

$$
\tilde{I}_{j}=e i \sum_{k} \mathcal{T}_{j}(x) e^{-i \alpha p} c_{j k}^{\dagger} d+h . c .
$$

with the total density matrix

$$
\tilde{\rho}(t)=e^{-i t\left(\tilde{\mathcal{H}}_{d o t}+\mathcal{H}_{\text {leads }}\right)} \tilde{\rho}_{V}(t) e^{i t\left(\tilde{\mathcal{H}}_{\text {dot }}+\mathcal{H}_{\text {leads }}\right)},
$$

where $\tilde{\rho}_{V}(t)$ obeys Eq. (17). Following the same steps and assumptions which we have made in the derivation of the rate equations, we obtain the average DC current flowing from the $j$ th lead to the dot as (we set $e=1$ )

$$
I_{j}=\sum_{n}\left(\Gamma_{j, n}^{<} P_{n}^{0}-\Gamma_{j, n}^{>} P_{n}^{1}\right)
$$

where $\Gamma_{j, n}^{<,>}$are the partial relaxation rates contributing to $\Gamma_{n}^{<,>}$, Eq. (28), from the $j$ th electrode:

$$
\begin{aligned}
\Gamma_{j, n}^{<} & =\sum_{m} \Gamma_{j} \gamma_{j, n m}^{2} f_{j}\left(\tilde{\epsilon}-\Omega_{n m}\right), \\
\Gamma_{j, n}^{>} & =\sum_{m} \Gamma_{j} \gamma_{j, m n}^{2}\left(1-f_{j}\left(\tilde{\epsilon}-\Omega_{m n}\right)\right) .
\end{aligned}
$$

By virtue of the rate equations, we have conservation of the current, $I_{l}=-I_{r}$. 


\section{PHONON-ASSISTED TRANSPORT FOR $\Gamma_{l} \ll \Gamma_{r}$}

In calculating $I(V)$-characteristics from Eqs. (18) and (36), we will focus on the case of highly asymmetric twojunction model with $\Gamma_{l} \ll \Gamma_{r}$. This corresponds to the typical experimental situation with STM measurements where a contact to the STM tip plays the role of the high resistive tunneling junction. For instance, in STM measurements on suspended nanotubes, the typical ratio $R_{t i p} / R_{s u b} \sim 10^{3}-10^{5}$ can be huge depending, in particular, on the tunneling distance between the STM-tip and the nanotube, $h \sim 4 \stackrel{\circ}{A}$ typically. In the present Section, no gate is present (floating level) thus $C_{g}=0$.

At the same time, according to the data in Ref. 8 obtained from spectroscopy measurements on suspended nanotubes, the ratio $C_{t i p} / C_{s u b} \equiv C_{l} / C_{r}$ can be smaller as well as larger than unity depending on an effective length of the portion of the nanotube that is on the substrate, and which can be relatively short due to local defects induced by the edges of the trench. Thus, there is no dominating asymmetry in the capacitive coupling of the dot to the both electrodes. As a result, in the voltage-biased system, the effective position of the polaron level with respect to the chemical potentials of the leads is strongly affected by the ratio $C_{l} / C_{r}$ which is not negligibly small like $\Gamma_{l} / \Gamma_{r}$, or can even be larger than unity ${ }^{30}$. It results that the polaron level (assuming neither gate-induced nor intrinsic shift of the polaron level at zero voltage) is not trivially stuck to the chemical potential of the far less resistive junction electrode, $\mu_{r}$.

Considering the system of stationary rate equations, Eq. (27), and writing explicitly expressions for the Fermi factors entering Eq. (29), we obtain [see also Eqs. (6), (7)]:

$$
f_{l}(\tilde{\epsilon})=f\left(E-c_{r} V\right), \quad f_{r}(\tilde{\epsilon})=f\left(E+c_{l} V\right), \quad V=\mu_{l}-\mu_{r}
$$

where $f(\omega)=\left(e^{\beta \omega}+1\right)^{-1}$, and

$$
E=\epsilon_{0}-g^{2} / \Omega+2 E_{C}\left(n_{x}+1 / 2\right)
$$

determines the position of the polaron level with respect to the lead chemical potentials at $V=0$, considered as the reference level of zero energy. From Eq. (39), the role of charging effects in the single-electron resonant level problem can be viewed as follows : when changing the bias voltage, the chemical potentials move in opposite directions (depending on the sign of $V$ ) with different "velocities" determined by the capacitance ratios, $c_{r}$ and $c_{l}$ for the left and right electrode, respectively. The above picture is given in the "reference frame" of the polaron level, where its position is voltage-independent. For the floating-dot geometry, $E$ may be strongly affected by a background charge, $n_{x}$. In the presence of a gate electrode ${ }^{30}, E$ can be controlled by the gate potential, and $C$ in Eq. (7), must also include the gate capacitance, with $c_{l}+c_{r}<1$.

In this section, in order to make analytical progress on the prediction of the current transport, we formally assume temperature to be zero. Because the rate equations have been derived in the high-temperature approximation $(T \gg$ $\left.\Gamma_{r}\right)$, the zero-temperature assumption is justified for the voltage-biased cases where the chemical potentials, $\mu_{l, r}$, are not very close to the phonon sidebands, so that thermally activated tunneling processes can be neglected.

We first notice that in the case where the polaron level $E$ lies beyond the bias-voltage window, the system of rate equations, Eq. (27), reduces to the following:

$$
L_{n m}^{<} P_{m}^{0}=L_{m n}^{>} P_{n}^{1},
$$

which is satisfied for all phonon numbers $m, n$. The ground state solution of Eq. (41) is either $P_{n}^{i}=\delta_{n 0} \delta_{i 1}(i=0,1)$ for $E<\mu_{l, r}$, or $P_{n}^{i}=\delta_{n 0} \delta_{i 0}$ for $E>\mu_{l, r}$. As a result, in this case the current is zero : no contribution to the current occurs from the phonon sidebands alone (cf. Ref. 16).

Eq. (41) also works in the less trivial case when $E$ lies within the bias-voltage window, but $\left|\mu_{l, r}-E\right|<\Omega$. For instance, for $V \equiv \mu_{l}-\mu_{r}>0$, the solution of Eq. (41) (valid for arbitrary $\Gamma_{l, r}$ ) reads $P_{n}^{i}=\delta_{n 0} P^{i}$, where

$$
\begin{aligned}
P^{0}=1-P^{1} & =\frac{L_{00}^{<}}{L_{00}^{<}+L_{00}^{>}}=\frac{\tilde{\Gamma}_{l}}{\tilde{\Gamma}_{l}+\Gamma_{r}}, \\
\tilde{\Gamma}_{l} & =\Gamma_{l} e^{-2 s \alpha+s^{2}}
\end{aligned}
$$

and the current is given by

$$
I=e^{-\alpha^{2}} \frac{\tilde{\Gamma}_{l} \Gamma_{r}}{\tilde{\Gamma}_{l}+\Gamma_{r}} .
$$

For large $\alpha$, the exponential prefactor in Eq. (44) leads to a significant suppression of the current at low bias voltages (the so-called Franck-Condon blockade $\left.{ }^{15,16,17}\right)$. The role of $s$ ("half-shuttle") will be discussed later. 
At higher voltages, such that phonon sidebands enter the bias-voltage window, Eq. (41) fails, and we have to go back to the full system of rate equations, Eq. (27), taking into account the total balance of probabilities for transitions between different states of the dot. This renders the problem of calculating phonon distribution functions, $P_{n}^{i}$, and eventually the $I(V)$-characteristics, a rather complicated one to be solved analytically. However, for the case of high asymmetry in the tunneling couplings, it is possible to make some further analytical progress before passing to numerical calculations 35 .

We now exploit the condition $\Gamma_{l} \ll \Gamma_{r}$ for analyzing steady solutions of the rate equations. In the general case, when a bias voltage $V$ is applied and the current is not identically zero (up to thermally activated processes), the polaron level is accompanied by $N+M$ phonon sidebands captured in the bias-voltage window, where we introduce ("int" stands for the integer part)

$$
\begin{aligned}
& N=\operatorname{int}\left(\left|\mu_{l}-E\right| / \Omega\right), \\
& M=\operatorname{int}\left(\left|\mu_{r}-E\right| / \Omega\right) .
\end{aligned}
$$

Independently of the sign of $V$, the integer $N(M)$ is defined as a number of phonon sidebands lying between the polaron level and the chemical potential of the electrode connected to the dot through the more (less) resistive junction. An example with $N=2$ and $M=1$ for $V>0$ is shown on Fig. 2] Due to high asymmetry $\Gamma_{l} \ll \Gamma_{r}$, only probabilities $\left\{P_{m}^{0}\right\}$ with $m \in[0, M]$ do not vanish in this limit. For the example on Fig. 2 the probabilities to have an electron on the dot with any $n$ phonons, $P_{n}^{1}$, are suppressed due to "fast" tunneling of the electron to the right electrode via $n+2$ open channels; the probability to have the dot with an empty electron state but with the number of phonons $M+1=2$ (and higher), $P_{M+1}^{0}$, is also negligible due to the "fast" tunneling of an electron from the right electrode with absorbing two phonons. In other words, on a large time scale determined by $\Gamma_{l}^{-1}$, the polaron-hole states with 0 and 1 phonon excitation are (quasi)steady states with respect to the tunneling coupling to the right electrode. Notice that in the asymmetric situation, the phonon number distributions are nearly the same in the polaron basis (the $P_{n}^{0}$ 's) and the original basis $\left(R_{p h}\right)$, as shown by Eq. (33).

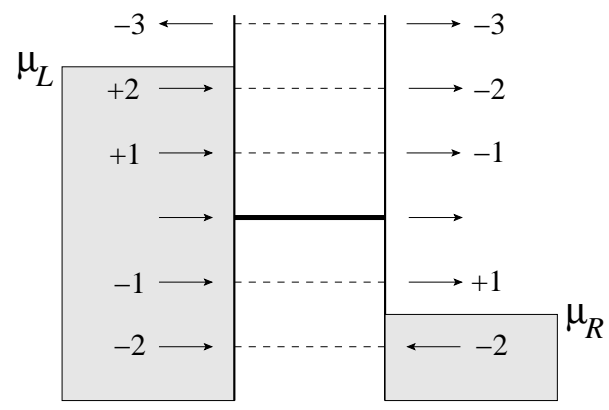

FIG. 2: Energy-level diagram for the case $M=1$ and $N=2$; the arrows show possible channels for electrons to tunnel onto/from the dot with changing (indicated by numbers) the phonon occupancy; $\mu_{l}-\mu_{r}=V$

The case $V<0$ can be treated in the same manner. We obtain that only probabilities $\left\{P_{m}^{1}\right\}$ with $m \in[0, M]$ are not vanishing as $\Gamma_{l} / \Gamma_{r}$. As a result, with a good accuracy, controlled by the smallness of $\Gamma_{l} / \Gamma_{r}$, the current flowing from the left to the right, $I \equiv I_{l}$ [see Eq. [36] ], can be written as

$$
I(V>0)=\sum_{m=0}^{M} P_{m}^{0} I_{m}, \quad I_{m}=\Gamma_{l} \sum_{n=0}^{m+N} \gamma_{l, m n}^{2},
$$

where $\sum_{m=0}^{M} P_{m}^{0}+O\left(\Gamma_{l} / \Gamma_{r}\right)=1$, and

$$
I(V<0)=-\sum_{m=0}^{M} P_{m}^{1} I_{m}, \quad I_{m}=\Gamma_{l} \sum_{n=0}^{m+N} \gamma_{l, n m}^{2},
$$

with $\sum_{m=0}^{M} P_{m}^{1}+O\left(\Gamma_{l} / \Gamma_{r}\right)=1$. The current is given by a sum of partial currents, $I_{m}$, representing $M$ conducting channels which are distributed with the corresponding phonon occupation probabilities. The magnitude of each partial current depends on the previously defined number of phonon sidebands $N$ captured between $E$ and $\mu_{l}$.

When varying $V$, the number of captured phonon sidebands, $N$ and $M$, can change. In the voltage ranges where $N$ and $M$ do not change, we obtain plateaus in the $I(V)$ characteristics. Assuming for concreteness $V>0$, we 
see from Eq. (47) that if $V$ increases in such a way that only $N$ changes ( $N$ can only increase with increasing $V$ ), the magnitude of each partial current, and hence the net current, will increase. Thus, we obtain here the positive differential conductance (PDC) behavior which is commonly computed and observed. On the contrary, if when increasing $V$, only $M$ changes (i.e. increases), while $N$ remains constant, then a new phonon-assisted channel is added. This leads to redistribution of phonon occupation probabilities between all open channels. Paradoxically, the net current may decrease, leading to a NDC behavior on $I(V))^{11}$.

For $V>0$, assuming that due to the voltage increase $V \rightarrow V+\Delta V$ the number of phonon sidebands in the bias-voltage window has changed as $\{N=0, M=0\} \rightarrow\{N=0, M=1\}$, using Eq. (47) the variation of the current $\Delta I=I(V+\Delta V)-I(V)$ becomes:

$$
\Delta I=P_{0}^{0} I_{0}+P_{1}^{0} I_{1}-I_{0}=-P_{1}^{0} \tilde{\Gamma}_{l} e^{-\alpha^{2}}\left[1-(\alpha-s)^{2}-\left(1-\alpha^{2}+s^{2}\right)^{2}\right] .
$$

Thus, for this particular situation, assuming for simplicity $s=0$, the condition for the NDC is $\alpha<1$ : large values of the electron-phonon coupling do not favor NDC. The magnitude of the negative step on $I(V)$ increases with increasing phonon occupancy $P_{1}^{0}$.

For $V<0$, considering the same transition, $\{N=0, M=0\} \rightarrow\{N=0, M=1\}$, one finds:

$$
\Delta I=-P_{1}^{1} \tilde{\Gamma}_{l} e^{-\alpha^{2}}\left[1-(\alpha+s)^{2}-\left(1-\alpha^{2}+s^{2}\right)^{2}\right],
$$

with the same NDC condition, $\alpha<1$, for $s=0$. From the above expressions, we also notice that for positive (negative) bias voltage and non-zero but small half-shuttle $s \ll 1$, the NDC effect increases (decreases) for the first phonon-assisted step of the $I(V)$ characteristic.

As far as the bias voltage increases, the current eventually saturates and does not change in practice. For large bias voltages $V \rightarrow \pm \infty$, the corresponding saturation currents $I_{\text {sat }}^{( \pm)}$are given by (up to corrections $O\left(\Gamma_{l}^{2} / \Gamma_{r}\right)$ ):

$$
\begin{aligned}
& I_{\text {sat }}^{(+)}=\Gamma_{l} \sum_{m=0}^{+\infty} P_{m}^{0}\left\langle m\left|e^{-2 s x}\right| m\right\rangle, \\
& I_{\text {sat }}^{(-)}=-\Gamma_{l} e^{-4 s \alpha} \sum_{m=0}^{+\infty} P_{m}^{1}\left\langle m\left|e^{-2 s x}\right| m\right\rangle .
\end{aligned}
$$

In the absence of the half-shuttle mechanism, we obtain that the saturation current does not depend on the phonon distribution, $I_{s a t}^{( \pm)}=\Gamma_{l}$. For $s \neq 0$, considering equilibrated phonons, i.e., forcing $P_{m}^{s} \rightarrow \delta_{m 0}$ in the limit of strong phonon relaxation, we obtain the saturation currents:

$$
I_{\text {sat }}^{(+)}=\Gamma_{l} e^{2 s^{2}}, \quad I_{\text {sat }}^{(-)}=-e^{-4 s \alpha} I_{\text {sat }}^{(+)} .
$$

For non-equilibrated phonons, Eq. (53) is expected to be accurate in the limit $c_{l} \ll c_{r}$. Nevertheless, for relatively small $\alpha<1$, the asymmetry in the current saturation values due to the half-shuttle can be noticeable at low bias voltages for $c_{l} \sim c_{r}$ because of the fast saturation of the partial currents $I_{m}$ (fast convergency of the series in Eqs. (47) and (48)).

\section{NUMERICAL RESULTS AND DISCUSSION}

We now turn to the numerical solution of the rate equations, and explore the parameter space in order to find the signatures of NDC. After writting the system of the rate equations, Eq. (27), in the matrix form, $\hat{\Lambda} P=0$, with $P^{T}=\left(P_{0}^{0}, P_{0}^{1}, P_{1}^{0}, P_{1}^{1}, \ldots\right)$, the problem reduces to finding the zero eigenvalue of the matrix $\hat{\Lambda} \underline{\underline{16}}$ The typical maximum number of phonon states has been taken around 40, so that results of the calculation would not depend on this choice for the considered range of parameters. From numerically found solutions $P$, the current has been calculated in the left and right electrodes using Eq. (36). The achieved tolerance for $I_{l}=-I_{r}$ was $10^{-6}$.

Contrary to the previous analytical arguments, calculations are performed at finite temperature. For calculation purposes we assume a large asymmetry in the tunneling rates, $\Gamma_{l} / \Gamma_{r}=10^{-4}$. Yet, most of our results also hold for moderate asymmetries. The current $I$ is plotted in units of $\Gamma_{l} \Gamma_{r} /\left(\Gamma_{l}+\Gamma_{r}\right)$, the bias voltage $V$, the polaron level $E$ and temperature $T$ are given in units of $\Omega$. In subsection $\mathrm{A}$, one considers the floating level case. We consider the effect of a given shift of the dot level (due to background or gate charges) in subsection B. In these two subsections we will disregard the half-shuttle $(s=0)$, which will be considered in the last subsection. 


\section{A. Non-shifted polaron level $(E=0)$}

We start by considering the case where the capacitances surrounding the dot fully specify the position of the polaron level. At zero bias voltage, the polaron level is aligned with the chemical potentials of the electrodes, $E=0$. In this case, as it immediately follows from the system of rate equations, Eq. (27), we have the symmetry relation $P_{n}^{0}(V)=P_{n}^{1}(-V)$ and consequently $I(-V)=-I(V)$. Notice that for the "floating-level" geometry the case of $E=0$ is an exceptional one, and must be viewed as a reference point.

Fig. 3 shows the $I(V)$ characteristics for different $\alpha$ 's assuming small $c_{l}=0.1$, so that the polaron level lies closer to $\mu_{r}$. In the experiment of Refs. 7 [30, this means that the nanotube has a large overlap with the conducting substrate. As long as $M=0$, that is if $V<\Omega / c_{l}$ (this condition is satisfied on the bias-voltage range which is plotted), $P_{n}^{0}=\delta_{n 0}-O\left(\Gamma_{l} / \Gamma_{r}\right)$, which results in the PDC behavior of $I(V)$. The PDC steps correspond to $N$ increasing by one each time $V$ passes through a multiple integer of $\Omega / c_{r}$. Explicitly, the current-step amplitude at $V=n \Omega / c_{r}$ corresponding to the current increase is given by

$$
\Delta I^{(n)}=\Gamma_{l} e^{-\alpha^{2}} \frac{\alpha^{2 n}}{n !} .
$$

For smaller $\alpha$ 's the current-step amplitude decreases faster with increasing $V$, which leads to the saturation of the current at lower voltages.

Note that the first step (low bias voltage) is rounded. This feature is specific to the fact that the capacitances are asymmetric. Indeed, increasing the bias voltage from zero, the chemical potentials move away from the polaron level, but the chemical potential whose lead has the largest capacitance remains close to this level. In this event, thermally activated tunneling processes can be effective. When the bias voltage is further increased the phonon sidebands which contribute to new steps are those above the polaron level, and thus their corresponding steps are not thermally rounded. In the case where the asymmetry in the capacitances is opposite, which is shown in the next figure, the same reasoning applies.

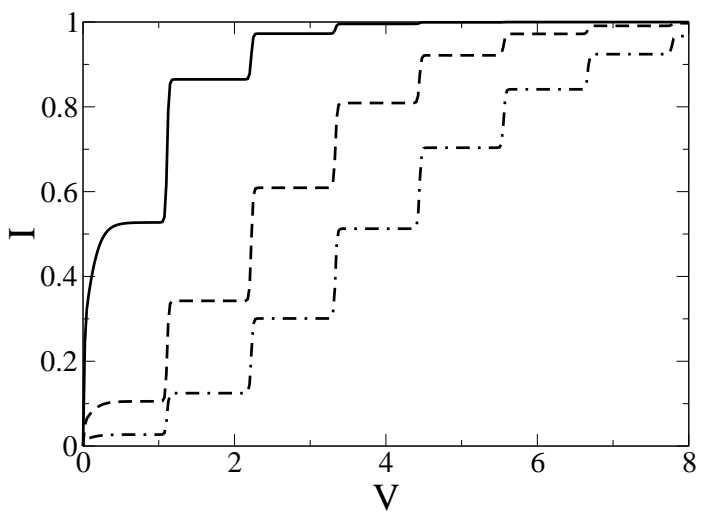

FIG. 3: The case $c_{l}=0.1$ for $\alpha=0.8$ (solid), 1.5 (dashed) and 1.9 (dot-dashed). $T=0.01$.

Fig. 4 shows the $I(V)$ characteristics for different $\alpha$ 's assuming a relatively large $c_{l}=0.9$. In the experiment of Refs. 730 , this means that the nanotube has a small length, thus a small overlap with the conducting substrate, and thus a small capacitance compared to that of the tip-nanotube contact. Alternatively, a large $c_{l}$ could be achieved if the nanotube contains impurities or bends.

In the bias-voltage window, the polaron level is closer to $\mu_{l}$ than to $\mu_{r}$. Within the bias-voltage range which is plotted, the current changes by steps when $V$ passes through a multiple integer of $\Omega / c_{l}$, as before. At these points, $M$ increases by one; correspondingly, one more phonon-assisted channel is added. For $\alpha=1.5$ one still observes the PDC behavior, although the height of the current steps is strongly suppressed compared to the previous case on Fig. 3] For $\alpha<1 \mathrm{NDC}$ occurs in the first step, and with decreasing $\alpha$, more NDC steps appear. For all curves, Eq.

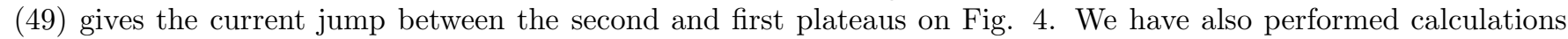
of the current voltage characteristics in this NDC regime while increasing gradually the temperature (not shown). Temperature tends to smooth the steps and to suppress NDC altogether when it becomes comparable to the spacing between the steps.

At the same time that we monitor the electronic current, it is instructive to examine the phonon occupation numbers in order to quantify PDC or NDC behavior. The phonon distributions $P_{m}^{0}$, which play the role of probabilities of

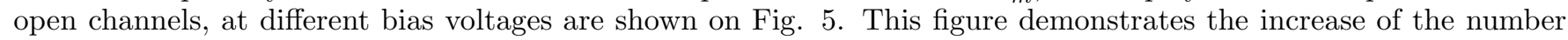




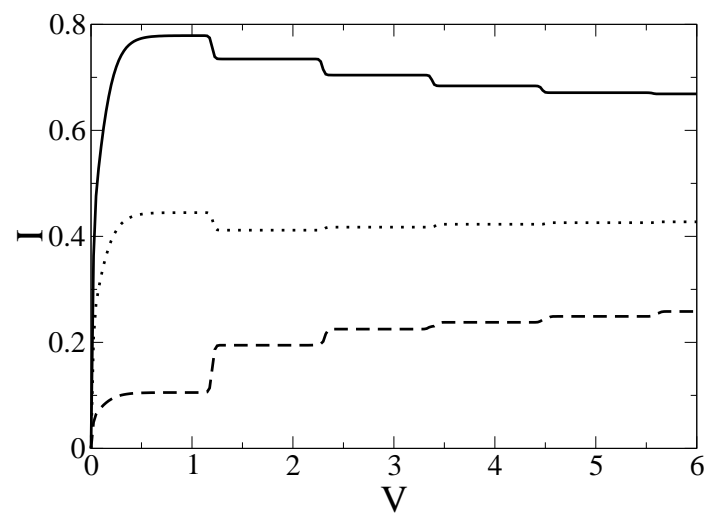

FIG. 4: The case $c_{l}=0.9$ for $\alpha=0.5$ (solid), 0.9 (dotted) and 1.5 (dashed). $T=0.01$.

of phonon excitations with increasing $M$ (See Eq. (46)). It shows that the phonon number which are occupied is restricted by $M-1$. Thus, the phonon distribution allows to understand the height of the PDC or the NDC steps.

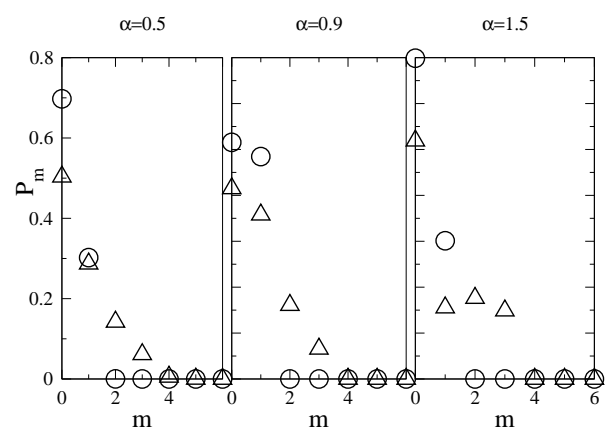

FIG. 5: Phonon distribution, $P_{m}^{0}$, for the case of Fig. 4 at the bias voltage $V=2$ (circles) and $V=4$ (triangles).

In the two previous cases, two limits were considered : either $c_{l} \ll c_{r}$ or $c_{l} \gg c_{r}$. On the way to the more general situation when $c_{l}$ and $c_{r}$ are comparable, let us now consider in more detail the capacitively symmetric case when $c_{l}=c_{r}=0.5$. The $I(V)$ characteristics for $\alpha=0.8$ and 1.5 are shown on Fig. 6. For $\alpha=1.5$, the current shows a

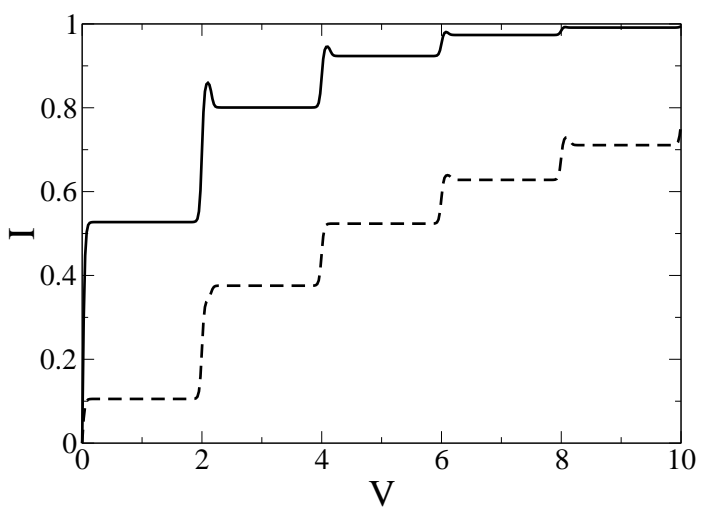

FIG. 6: The case $c_{l}=0.5$ for $\alpha=0.8$ (solid) and 1.5 (dashed). $T=0.01$.

stepwise increased with essentially no NDC behavior. Note that because the capacitances are equilibrated, the first step does not display substantial rounding due to thermal effects. For $\alpha=0.8$, one observes a stepwise increase of the current, but the onset of each step is associated with a small spike, thus exhibiting NDC behavior. This NDC behavior is thus qualitatively different from the one previously observed for the case of asymmetric capacitances. The spikes bringing the NDC features to the $I(V)$ characteristics are robust with respect to temperature. In Fig. 7 one sees that 
these spikes are broadened with temperature, but NDC persists as long as the temperature is not comparable to the phonon frequency. On the $I(V)$ for $\alpha=1.5$ (Fig. (6), the NDC-singularities appear only for higher voltages, however, the first phonon-assisted current step (at $V=2 \Omega$ ) is also deformed.

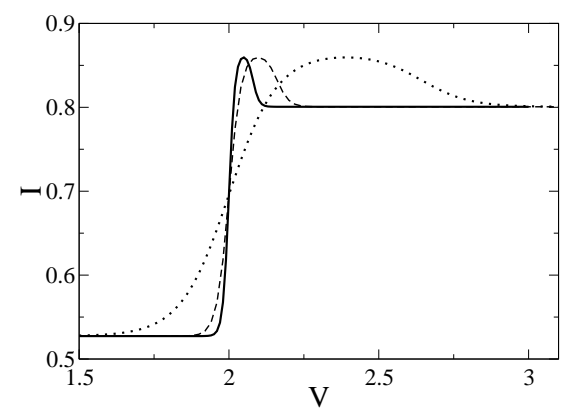

FIG. 7: Temperature dependence of the NDC-singularity for $\alpha=0.8: T=0.005$ (solid), 0.01 (dashed) and 0.04 (dotted).

At low temperature, it is interesting to make a zoom of a given step in order to see how the spikes evolve when the electron phonon coupling is varied. Fig. [8] shows these "singularities" on the second step of the $I(V)$ characteristic for a wide range of $\alpha$ 's (cf. Fig. 6). Note that the NDC behavior is more pronounced with increasing $\alpha=\{0.5,0.8,1.1\}$. Indeed, in the limit of vanishing electron phonon coupling, one gets only one step due to the polaron (electron) level, and all NDC features are absent. There is therefore an optimal value of $\alpha$ which displays maximal NDC behavior. Further increasing $\alpha$, NDC converts to the PDC case (the curves for $\alpha=\{1.5,1.9\}$ ).

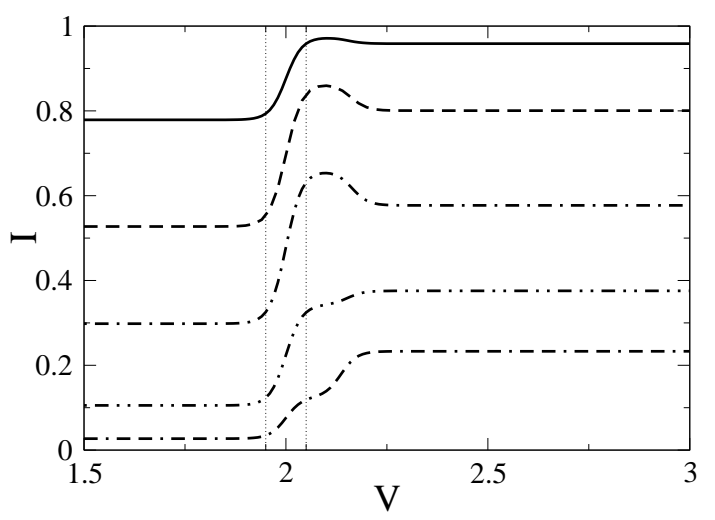

FIG. 8: From up to down $\alpha=\{0.5,0.8,1.1,1.5,1.9\}$ for $c_{l}=0.5 . \quad T=0.01$. The dotted vertical lines around $V=2$ (corresponding to $V_{ \pm}=2 \pm 0.05$ ) show the width of the Fermi distribution function at given $T$.

In this case of equal capacitances $\left(c_{l}=c_{r}\right)$, the polaron level lies in the middle of the bias-voltage window at all $V$, so that both $N$ and $M$ increase by one when $V$ passes through a multiple integer of $2 \Omega$. In particular, at $V=2 \Omega$ the transition $\{N=M=0\} \rightarrow\{N=M=1\}$ occurs : below $V=2 \Omega$ the polaron level is the only one which lies within the bias voltage window, while immediately above $V=2 \Omega$, two phonon sidebands are simultaneously captured by the bias window. As a result, in the vicinity of $V=2 \Omega$ (how close depends precisely on temperature), we have a competition between the PDC- and "possible NDC"-type contributions to the current, corresponding to the processes of electron tunneling from the left electrode into the dot with emission and absorption of one phonon, respectively.

In the transition region, $V \approx 2 \Omega$, the current is given by (here we take into account the Fermi factors)

$$
\begin{gathered}
I(V)=\sum_{m=0}^{1} P_{m}^{0}(V) I_{m}, \\
I_{m}=\Gamma_{l} \sum_{n=0}^{m+1} \gamma_{l, m n}^{2} f\left(-c_{r} V-\Omega_{m n}\right),
\end{gathered}
$$


and $P_{0}^{0}+P_{1}^{0}+O\left(\Gamma_{l} / \Gamma_{r}\right)=1$. Explicitly, we have

$$
I(V)=\Gamma_{l} e^{-\alpha^{2}}\left[1+\alpha^{2} f(\Omega-V / 2)+P_{1}^{0}(V) \alpha^{2}\left(-1+\alpha^{2}+\frac{1}{2}\left[\left(1-\alpha^{2}\right)\left(2-\alpha^{2}\right)-\alpha^{2}\right] f(\Omega-V / 2)\right)\right] .
$$

¿From the numerics, it follows that up to $V=V_{+} \equiv 2 \Omega+W>0$, where $W$ is the half-width of the Fermi distribution function (see caption of Fig. 8), we have $P_{1}^{0}(V)=0$, thus the current successively increases up to:

$$
I\left(V_{+}\right)=\Gamma_{l} e^{-\alpha^{2}}\left(1+\alpha^{2}\right) .
$$

This effect is due to the high asymmetry in the tunneling rates $\Gamma_{l, r}$ : the probability to have an empty dot with one phonon, $P_{1}^{0}$, remains negligible, $O\left(\Gamma_{l} / \Gamma_{r}\right)$, until there is some probability to have thermally activated electrons of energy $E-\Omega$ coming from the right electrode. Only then, for $V>V_{+}$, where $f(\Omega-V / 2)$ has practically reached unity, $P_{1}^{0}(V)$ starts deviating from zero. Thus, we have

$$
\begin{gathered}
I(V)-I\left(V_{+}\right)=P_{1}^{0}(V) \Gamma_{l} e^{-\alpha^{2}} g(\alpha), \quad V>V_{+}, \\
g(\alpha)=\alpha^{2}\left(-1+\alpha^{2}+\frac{1}{2}\left[\left(1-\alpha^{2}\right)\left(2-\alpha^{2}\right)-\alpha^{2}\right]\right) .
\end{gathered}
$$

For $V>V_{+}$, the $V$-dependence of the current is given by $P_{1}^{0}(V)$. With increasing $V, P_{1}^{0}(V)$ also increases and

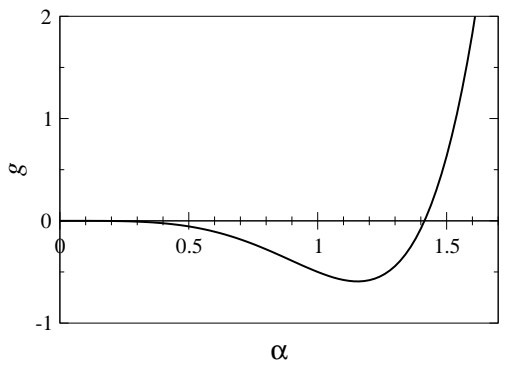

FIG. 9: $g(\alpha)$ function, Eq. (60).

saturates to some quantity (depending on $\alpha$ ) which is comparable with $P_{0}^{0}$ (the right-hand side plateau on Fig. 8). From numerics we find that at $V=2.5 \Omega$, for instance, for $\alpha=\{0.5,1.1,1.9\}$, we correspondingly have $P_{1}^{0} \approx 1-P_{0}^{0}=$ $\{0.35,0.48,0.38\}$. From Eq. (59) and Fig. 9] we see that the NDC plateau disappears for small $\alpha<0.5$; with increasing $\alpha$, the NDC contribution from an open channel increases-decreases having the maximum at $\alpha \approx 1.15$, and for $\alpha>1.4$ the NDC contribution converts to the PDC one (cf. Fig. 8).

Up to now, we have considered only strong asymmetric capacitances and equal capacitances. A common feature of these choices is that the current voltage characteristics displays steps (with or without NDC behavior) whose spacing in voltage is essentially always the same at relatively low voltages. Indeed, for the asymmetric capacitances plots each step is separated by (approximately) $\Omega$, while for $c_{l}=c_{r}$, this spacing is $2 \Omega$. If one was to plot the differential conductance, as in the experiment of Ref. 7, one would obtain a periodic series of peaks. What happens to this periodicity in an intermediate situation where the capacitances are comparable, but not equal ? Fig. [10 shows current-voltage characteristics with two steps of different length for the case $c_{r}=1.5 c_{l}$. The number of phonon sidebands above the polaron level, $N$, increases by one with the voltage period $\Omega / c_{r}=(5 / 3) \Omega$; for these steps we always have a PDC behavior as it is demonstrated in the previous section. At the same time, the number of phonon sidebands below the polaron level $M \rightarrow M+1$ with the voltage period $\Omega / c_{l}=2.5 \Omega$ : one more channel becomes open which yields the possibility of NDC. The larger $\alpha$, the more PDC-steps at low bias voltages are observed.

\section{B. Shifted polaron level $(E \neq 0)$}

In this subsection, we consider the more general situation where, due to gate or background charges, the polaron level $E$ is shifted by a constant value with respect to the chemical potentials of the leads at zero bias voltage. For $E \neq 0$, the symmetry $I(V)=-I(-V)$ is violated, moreover $I(V)$ is not shifted in a trivial manner, meaning that it does not simply follow $E$. Fig. 11] shows two current-voltage characteristics for different values of $E$. As it was 


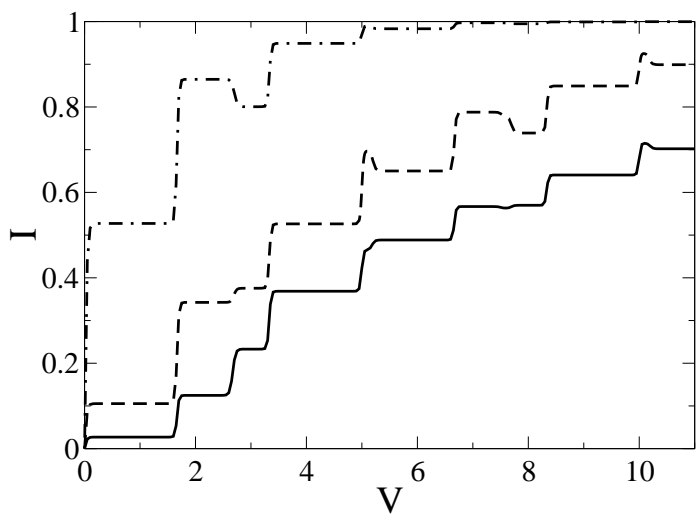

FIG. 10: $\alpha=1.9$ (solid), 1.5 (dashed) and 0.8 (dot-dashed) for $c_{l}=0.4, E=0, T=0.01, s=0$.

mentioned in Sec. IV, the current is zero when the polaron level lies beyond the bias voltage window. For negative $V$, the polaron level becomes captured in this window at $V=-E / c_{l}=-4 E$. At this point, the number of phonon subbands below the bare level $-N$ in this case - suddenly changes from $N=0$ to $N=\operatorname{int}\left(c_{r} E / c_{l} \Omega\right)=\operatorname{int}(3 E / \Omega)$. In the curves of Fig. [1] corresponding to $E=0.4 \Omega$ and $0.7 \Omega$, we observe a transition to $N=1$ and $N=2$, respectively. This results in a fast saturation of the curves at negative voltages (the saturation is faster for the curve with larger $E)$.

Previously, we found that small $c_{l}$ leads to PDC for the first phonon assisted peaks. Here, although $c_{l}=0.25$ is relatively small in Fig. 11] for positive $V$, we observe NDC behavior already for the first phonon-assisted step on $I(V)$ (around $V=2 \Omega$ ) for $E=0.7$. This can be explained as follows. At small positive voltages, we first have a zero-current plateau until the polaron level becomes captured in the bias-voltage window $(N=M=0)$ at $V_{0}=E / c_{r}$. This leads to the first PDC step on the $I(V)$ curve at $V_{0}=(4 / 3) E$. Then the second plateau persists until $V_{1}=(\Omega-E) / c_{l}<2 \Omega$, which corresponds to the phonon sideband level $E-\Omega$ becoming captured in the bias window $(N=0, M=1)$. Because $\alpha=0.8$ is less than the critical value 1 (see Sec. IV), this transition gives the NDC step. Next a plateau persists until, $V_{2}=(\Omega+E) / c_{r}$ when the phonon sideband level $E+\Omega$ is captured in the bias window $(N=1, M=1)$ resulting in a PDC step on $I(V)$. The conditions to observe NDC for the first phonon-assisted step are therefore:

$$
c_{r} \Omega>E>\left(c_{r}-c_{l}\right) \Omega>0, \quad \alpha<1 .
$$

The width of the NDC plateau is $V_{2}-V_{1}=\left(E-\left(c_{r}-c_{l}\right) \Omega\right) / c_{l} c_{r}$. Eq. (61) is fulfilled for the case $E=0.7 \Omega$ on Fig. [1] but it fails for $E=0.4 \Omega$, where we have the PDC behavior of the first phonon-assisted step on $I(V)$, for $V>0$.

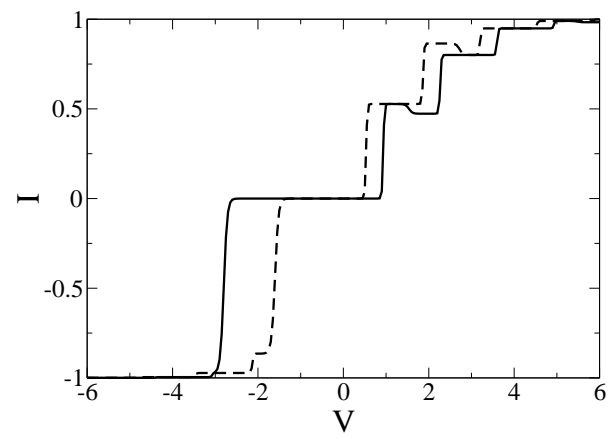

FIG. 11: $E=0.4$ (dashed) and $E=0.7$ (solid) for $\alpha=0.8, T=0.01, c_{l}=0.25$.

Fig. 12 shows the temperature dependence of the NDC step for $E=0.7 \Omega$. With increasing temperature $T$, the phonon-assisted channel opens for higher voltages when the one-phonon excited state of the empty dot becomes unreachable for thermally activated electrons from the right electrode at energy $E-\Omega+W$, (as before, $W$ is the half-width of the Fermi function). As a result, the NDC region with a decreased slope gradually shifts towards the next step on $I(V)$.

Fig. 13 shows the opposite situation: the NDC appears this time with increasing temperature. At low temperature, when the Fermi half-width $W$ is negligibly small, we only observe PDC behavior for the first step on $I(V)$ at $V_{0}=E / c_{r}$, 


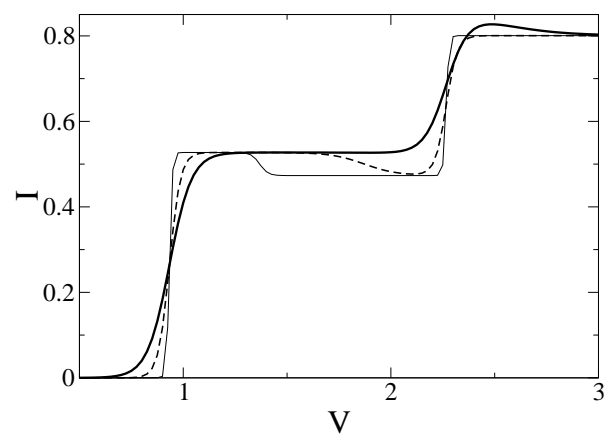

FIG. 12: The case $\alpha=0.8, c_{l}=0.25, E=0.7$ at different temperatures: $\mathrm{T}=0.01$ (thin solid), 0.02 (dashed) and 0.04 (bold solid).

where we suddenly have a transition to $\{N=0, M=1\}$. At this threshold, both the polaron level and the first phonon subband below it belong to the bias voltage window. Then we obtain a plateau in the $I(V)$ curve up to $V_{1}=(E+\Omega) / c_{r}$, where the next PDC step is associated with the increase of $N$ by one. However, if the temperature is increased so that $W$ becomes comparable with $\Omega-E$, now at $V=V_{0}$ the occupation probability $P_{1}^{0}$ will be negligibly small because of thermally activated electrons tunneling from the right electrode. $P_{1}^{0}$ starts developing only at $V>(\Omega-E+W) / c_{l} \approx 1.2 \Omega$ leading to NDC behavior. Here we have used that at $T=0.04 \Omega$, the half-width of the Fermi function is about $0.2 \Omega$. At much higher temperature everything is of course washed out (not shown).

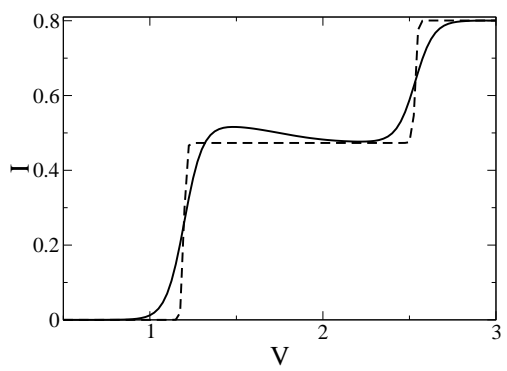

FIG. 13: The NDC induced by temperature for the case $\alpha=0.8, c_{l}=0.25, E=0.9: T=0.005$ (dashed) and 0.04 (solid).

We conclude this subsection by some comments on the role of the asymmetry in the tunneling rates $\Gamma$ 's on current transport. Fig. 14 shows $I(V)$ characteristics for the different cases of junction asymmetry. As it is seen from the plots, the NDC steps in the cases of high and moderate asymmetry $\left(\Gamma_{l} / \Gamma_{r}=0.01\right.$ and 0.1 , respectively) turn into PDC steps in the symmetric case $\left(\Gamma_{l}=\Gamma_{r}\right)$. We also notice that the NDC steps are slightly shifted with respect to their PDC counterparts in the symmetric case. This shift is associated with the finite width of the Fermi function, and it has been already been discussed in the two previous figures. The height of the phonon-assisted steps in the

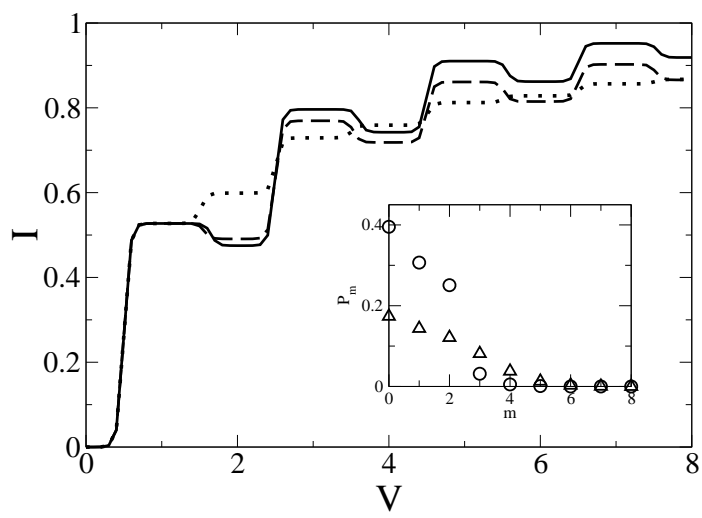

FIG. 14: For $\alpha=0.8, T=0.02, E=0.25, c_{l}=0.5: \Gamma_{l} / \Gamma_{r}=10^{-2}$ (solid), 0.1 (dashed) and 1 (dotted). The inset shows the phonon distribution $P_{m}^{0}$ at the bias voltage $V=4$ : circles for $\Gamma_{l} / \Gamma_{r}=10^{-2}$ and triangles for $\Gamma_{l} / \Gamma_{r}=1$. 
asymmetric case is noticeably large compared to the symmetric one. This is related to the phonon distribution, an example of which (at $V=4 \Omega$ ) is shown on Fig. 14 for both cases (symmetric and asymmetric). In the asymmetric case, the number of phonons is restricted by $M$, while in the symmetric case the phonon distribution is more spread out. As a result, in the symmetric case, the phonon-assisted contribution to the current is weakened.

\section{Effect of the half-shuttle on $I(V)$}

In this subsection we discuss the role of the half-shuttle mechanism on $I(V)$ characteristics.

It is worth to be mentioned that in the absence of the half-shuttle $(s=0)$, the rate equations (27) are invariant under the transformation

$$
V \rightarrow-V, \quad E \rightarrow-E, \quad P_{n}^{0} \leftrightarrow P_{n}^{1} .
$$

As a consequence, the current-voltage characteristics $I_{E}(V)$ and $I_{-E}(V)$ corresponding to the cases of the polaron level shifted by $E$ and $-E$, respectively, are related as follows:

$$
I_{E}(V)=-I_{-E}(-V) .
$$

The presence of half-shuttle mechanism $(s \neq 0)$ violates this symmetry.

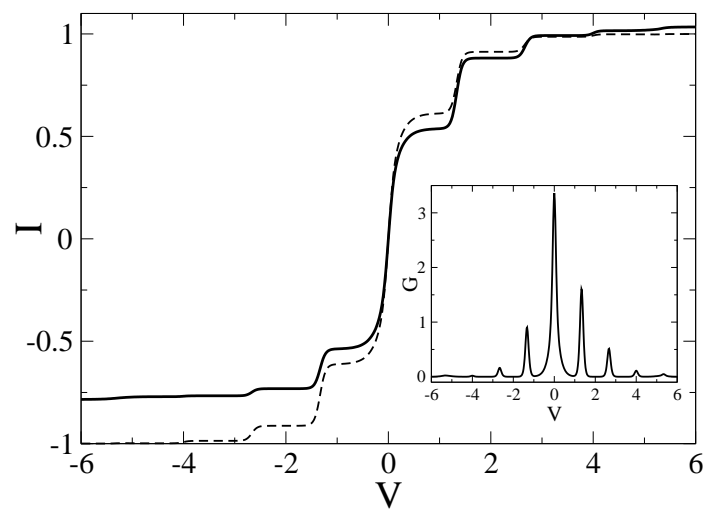

FIG. 15: Asymmetry of $I(V)$ due to "half-shuttle" only: $s=0.1$ (solid) and $s=0$ (dashed). The case of $\alpha=0.7, c_{l}=0.25$, $E=0, T=0.04$. The inset shows the differential conductance for $s=0.1$.

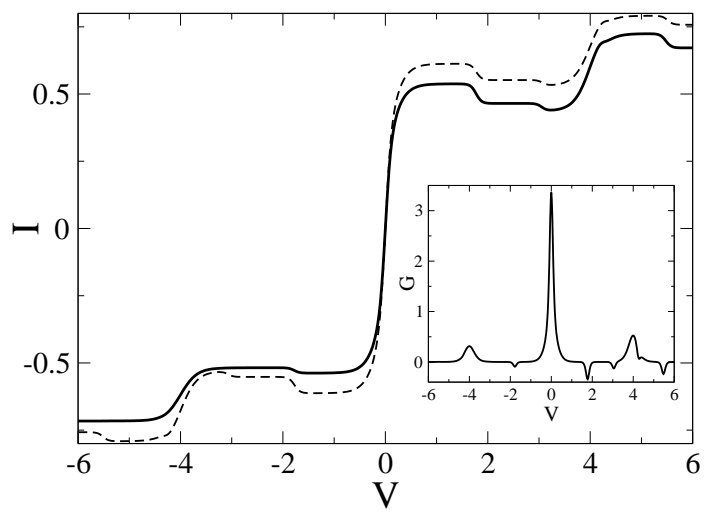

FIG. 16: Asymmetry of $I(V)$ due to "half-shuttle": $s=0.1$ (solid) and $s=0$ (dashed). The case of $\alpha=0.7, c_{l}=0.75, E=0$, $T=0.04$. The inset shows the differential conductance for $s=0.1$.

Fig. [15] shows $I(V)$ for the case when $c_{l}=0.25$ is relatively small and where the polaron level is not shifted, both of which are favorable for PDC. The current steps are suppressed at negative voltages (differential conductance peaks 
in the inset of Fig. 15), while at positive bias voltage, the current steps have a tendency to increase. From Eqs. (47), (48) we obtain that the current-step amplitude at $V=n \Omega / c_{r}$ corresponding to the current increase is given by

$$
\Delta I^{(n)}=\Gamma_{l} e^{-\alpha^{2}-2 s \alpha+s^{2}} \frac{(\alpha+\operatorname{sign}(V) s)^{2 n}}{n !} .
$$

In other words, the half-shuttle mechanism works in favor of the formation of the polaron state: the probability of phonon-assisted tunneling onto the dot from the left electrode is increased, while the phonon-assisted tunneling from the dot to the left electrode is decreased. Eq. (53) for the saturation currents illustrates this fact.

The case of relatively large $c_{l}=0.75$ is shown on Fig. 16] Like in the previous case, here we also observe suppression of the current steps at negative voltages. At positive voltages, the NDC steps become more pronounced compared to the PDC ones. Expressions for the one-phonon assisted steps are given by Eqs. (49), (50).

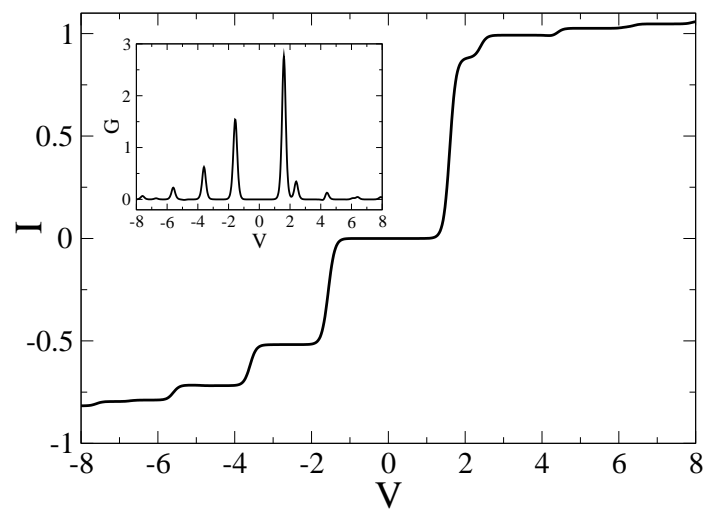

FIG. 17: The case $\alpha=0.7, c_{l}=0.5, E=-0.8, T=0.04, s=0.1$.

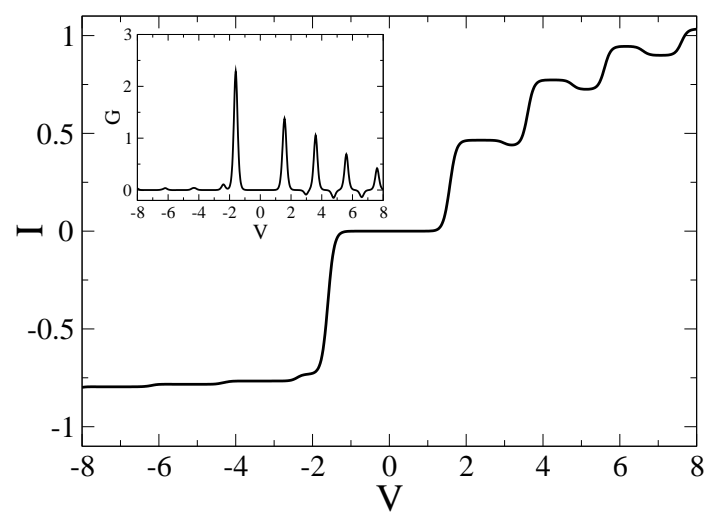

FIG. 18: The case $\alpha=0.7, c_{l}=0.5, E=0.8, T=0.04, s=0.1$.

We have provided concrete evidence that the half-shuttle mechanism alone, with reasonable values of the parameters, cannot produce NDC when $c_{l}$ is small and when the bare polaron level is not shifted upwards. Nevertheless, based on the results of Figs. 17 and 18 we conclude that in situation when NDC is present (shifted level or reversed capacitances), the addition of the half-shuttle tends to emphasize the NDC features.

\section{CONCLUSION}

To summarize, we have provided an in-depth study of NDC behavior in molecular quantum dots or transistors. While this study covers a wide range of experimental parameters such as capacitances and electron-phonon coupling, here the focus was put on the case where the tunneling coupling from the dot to the source and drain leads is 
asymmetrical. This choice was motivated by the experiment of Leroy and coworkers 7.30 where a nanotube is suspended over a trench, allowing nearly free vibrations of the phonon breathing mode. Electrons flow from an STM tip to the suspended region of the nanotube, and their tunneling amplitude is much smaller than that of the nanotube to the substrate. It turns out that this assumption allows us to quantify NDC behavior by analytical means. Yet, moderate asymmetries, often encountered with two poor metallic contacts, qualitatively display the same physics.

We have used a microscopic approach modeling the molecule/nanotube as a quantum dot, which is justified based on the early observation of Coulomb blockade behavior in carbon nanotubes. A single phonon mode couples on-site to the quantum dot, in two different way. First, as the principal mechanism, it couples to the electron density on the dot, as in a polaron model. Second, the half-shuttle coupling was introduced, with the motivation that it should be present in the experiment of Refs. 7.30: when the nanotube vibrates, the tunneling distance between the nanotube wall and the STM tip oscillates accordingly, but the amplitude of this motion is believed to be rather small (or the order of the zero point motion, which can be smaller than $0.1 \stackrel{\circ}{A}$ ).

With these ingredients, the polaron transformation eliminates the electron charge coupling to the phonon on the dot, and transfers this coupling to the tunneling Hamiltonian. At this point, we have chosen to describe the situation corresponding once again to the experiment of Ref. 7 30, where the time scale associated with tunneling events between the dot and the leads is large compared to the temperature, so that electrons evacuated in the leads effectively loose their phase coherence. On the other hand, the quantum mechanical nature of electron-phonon dynamics within the dot (polaron formation) is fully captured. This happens to be the regime were most molecular electronics experiments are performed nowadays, as these experiments typically do not require dilution refrigerator technology. From the density matrix of the total system, a kinetic equation for the reduced density matrix (with the leads degrees of freedom integrated out) was derived, leading to master equations for the electron population and their associated phonon numbers.

At temperature high enough for this rate equation is valid, but low enough that thermally activated tunneling from the phonon sideband can be neglected, analytical predictions were made for the current-voltage characteristics, thanks to our assumption of a highly asymmetrical molecule-lead configuration: the positive or negative differential conductance behavior depends both on the location of the bare polaron level and on the occupation of the associated phonon sidebands - above and below this level - which are included in the bias voltage window.

Turning to numerics, attention was first drawn to the role of the relative capacitances to the dot. If the molecule/nanotube is in better contact with the lead which evacuates the electrons (the substrate), than with the one who injects electrons, it is plausible to believe that the capacitance of this junction will be larger than that of the injecting junction. In this case, no negative differential conductance is obtained. When increasing the bias voltage, the contribution of an increasing number of phonon sidebands leads to a standard staircase behavior in the current-voltage characteristics. Thermal effects tend to round off the first step. In the experiment of Ref. 7 .30, the use of several nanotube samples with different lengths allowed to vary the relative capacitance of the injecting and of the tunneling contact. This provided a motivation to study the so-called "reversed capacitance" case, which according to our analytical prediction could justify the presence of an negative differential behavior. This was indeed observed numerically, but it was emphasized that intermediate phonon couplings are needed to observe NDC: "small" electron-phonon coupling leads to barely noticeable NDC, while "large" electron-phonon coupling suppresses NDC altogether, thus the need to use non-perturbative techniques to study NDC.

Do these results disqualify the possibility of NDC if contacts where the capacitances between left and right are comparable? The answer is no, although NDC could be more difficult to detect in this case. While the global shape of the current voltage characteristics displays the staircase structure typically attributed to PDC, the onset of each step is followed by a small spike, thus displaying NDC behavior. The amplitude of this peak can be optimized with phonon coupling. For slightly asymmetric capacitances, the same effects are observed, but the staircase structure is modified as it involves two distinct periodicities.

Because background charges may shift the bare polaron level, and because of the possibility of using a voltage gate on the molecule/dot we also studied the possibility of NDC in this case. This suggests that when the level is shifted upwards, NDC is possible even when the capacitance of the injecting junction is small.

Finally, we have proposed that a half-shuttle mechanism may play a role in a STM experiment. It can be detected by the asymmetry of the current-voltage curves. Yet, in our opinion, this mechanism cannot account for NDC alone and only tends to increase the heights of PDC and NDC steps in the current-voltage characteristics. Notice that approaching the tip closer to the molecule should strongly enhance the half-shuttle mechanism. In our numerical calculations, we set $s=0.1$ assuming the amplitude of the zero-point RBM oscillations to be of the order of 1 pm. Estimating the zero-point amplitude, we have considered a single-wall carbon nanotube as an elastic hollow cylinder with diameter $d_{0} \sim 1 \mathrm{~nm}$, with wall thickness $\sim 1 \stackrel{\AA}{A}$, and with a length corresponding to the relevant breathing part $\sim 10 \mathrm{~nm}$. Accordingly, the undeformed volume of the cylinder is $V_{0} \sim 1 \mathrm{~nm}^{3}$. The elastic properties of the nanotube cylinder are characterized with the average Young's modulus $Y \sim 1 \mathrm{TPa}$ (see, for instance, Ref. 37). Assuming $\hbar \Omega \approx 10 \mathrm{meV}$ ( $\Omega$ is the frequency of the RBM), one finds for the relative extension of the nanotube due to the 
breathing,

$$
\delta x \sim\left(\frac{\hbar \Omega}{Y V_{0}}\right)^{1 / 2} \sim 10^{-3} .
$$

Thus, the estimated diameter change, $\sim \delta x d_{0}$, associated with the zero-point oscillations is of the order of 1 pm.

Overall, we have provided a rather complete account on the possible occurrence of NDC behavior in molecular electronic transport, due to phonon-assisted transport. This phenomenon is due to a distribution of the total spectral weight of the injected electron on the polaron levels included in the bias window. Taking into account the correct microscopic expressions of the Franck-Condon factors associated to phonon transitions, we have demonstrated that NDC effects are a fingerprint of the polaronic nature of charge carriers. While we cannot rule out that NDC could exist in molecular system with symmetric molecular junction, it was not observed within this model. On the other hand, we have clearly shown that for asymmetric tunneling rates, there exist a wide range of parameters which lead to NDC.

A striking result of this work is the fact that a simple counting of the NDC steps in the current voltage characteristic (or alternatively, a counting of the peaks in differential conductance) provides direct information about how many phonon subbands contribute to transport. The question remains about whether this NDC behavior can be observed in experiments. Our model could apply to Ref. 6, if the tunnel couplings are indeed asymmetric. Concerning Ref. 7. NDC occurs but only for the first phonon sideband. This can be due to several factors : first, as a general rule, the height of PDC or NDC steps tends to decrease with increasing phonon sidebands number. Furthermore, if the electron phonon coupling is weak, NDC is present but its manifestation is weak. Finite temperature tends to smear these steps, so in order to observe several peaks in NDC, lower temperatures would be required, keeping in mind that the tunneling contacts should not be too large in order to "avoid" any Kondo like regime. Another aspect which we have not considered in this work is the possibility of phonon damping. The population of the phonon subbands may rearrange due to the coupling with the environment of the molecule ${ }^{15.16}$. The environment could include electron-hole pairs generated in the leads, which should be reduced because of our weak coupling assumption. On the other hand, for a "large" molecular system, the optical phonon mode (the breathing mode of the nanotube of Ref. 7) is also accompanied by other phonon modes such as acoustic-like modes, which have typically lower energies, and which therefore can play the role of a "bath". Because of the importance of the occupation of the phonon subbands for NDC in our present work, we expect that substantial coupling to this bath will tend to suppress NDC.

\section{Acknowledgments}

Discussions with V. Mujica and H. van der Zant are gratefully acknowledged.

${ }^{1}$ H. Park, J. Park, A.K.L. Lim, E. H. Andersen, A.P. Alivisatos, and P.L. McEuen, Nature (London) 407, 57 (2000).

2 N.B. Zhitenev, H. Meng, and Z. Bao, Phys. Rev. Lett. 88, 226801 (2002).

${ }^{3}$ L.H. Yu and D. Natelson, Nanolett. 4, 79 (2004); L.H. Yu, Z.K. Keane, J.W. Ciszek, L. Cheng, M.P. Stewart, J.M. Tour, and D. Natelson, Phys. Rev. Lett. 93, 266802 (2004).

4 X.H. Qiu, G.V. Nazin, and W. Ho, Phys. Rev. Lett. 92, 206102 (2004).

5 A.N. Pasupathy, J. Park, C. Chang, A.V. Soldatov, S. Lebedkin, R.C. Bialczak, J.E. Grose, L.A.K. Donev, J.P. Sethna, D.C. Ralph, and P.L. McEuen, NanoLetters 5, 203 (2005).

6 S. Sapmaz, P. Jarillo-Herrero, Ya.M. Blanter, C. Dekker, and H.S.J. van der Zant, cond-mat/0508270 (unpublished).

7 B. J. LeRoy, S. G. Lemay, J. Kong, and C. Dekker, Nature 432, 371 (2004).

8 B. J. LeRoy, J. Kong, V. K. Pahilwani, C. Dekker, and S. G. Lemay, Phys. Rev. B 72, 075413 (2005).

9 M.S. Dresselhaus, and P.C. Eklund, Adv. Phys. 49, 705 (2000).

10 V. Perebeinos, J. Tersoff and P. Avouris, Phys. Rev. Lett. 94,086802 (2005).

11 D. Boese and N. Schoeller, Eur. Phys. Lett. 54, 668 (2001).

12 U. Lundin and H. McKenzie, Phys. Rev. B 66, 075303 (2002).

13 K. D. McCarthy, N. Prokof'ev and M. T. Tuominen, Phys. Rev. B 67, 245415 (2003).

14 V. Aji, J.E. Moore, and C.M. Varma, Int. J. Nanosci. 3, 255 (2004).

15 K. Flensberg, Phys. Rev. B 68, 205323 (2003); S. Braig and K. Flensberg, Phys. Rev. B 68, 205324 (2003).

16 A. Mitra, I. Aleiner, and A. J. Millis, Phys. Rev. B 69, 245302 (2004).

17 J. Koch and F. von Oppen, Phys. Rev. Lett. 94,206804 (2005); J. Koch, M. Semmelhack, F. von Oppen, and A. Nitzan, cond-mat/0504095 (unpublished).

18 K. C. Nowack and M. R. Wegewijs, cond-mat/0506552 (unpublished) 
19 G.A. Kaat and K. Flensberg, Phys. Rev. B 71, 155408 (2005).

20 M.H. Hettler, H. Schoeller, and W. Wenzel, Europhys. Lett. 57, 571 (2002).

21 M. Galperin, M.A. Ratner and A. Nitzan, NanoLett. 5, 125 (2005).

22 S. Datta, Nanotechnology 15, S433 (2004).

23 L.I. Glazman and R.I. Shekhter, Zh. Eksp. Teor. Fiz. 94, 292 (1988) [Sov. Phys.-JETP 67, 163 (1988)]; N.S. Wingreen, K. W. Jacobsen, and J. W. Wilkins, Phys. Rev. Lett. 61, 1396 (1988). ibid., Phys. Rev. B 40, 11834 (1989).

24 H. Ness and A. J. Fisher, Phys. Rev. Lett. 83, 452 (1999).

25 E.G. Emberly and G. Kirczenow, Phys. Rev. B 61, 5740 (2000).

26 J. Bonca and S.A. Trugman, Phys. Rev. Lett. 75, 2566 (1995); ibid., Phys. Rev. Lett. 79, 4874 (1997).

27 Y. Meir and N. S. Wingreen, Phys. Rev. Lett. 68, 2512 (1992).

28 Jian-Xin Zhu and A. V. Balatsky, Phys. Rev. B 67, 165326 (2003).

29 A.N. Pasupathy, R.C. Bialczak, J. Martinek, J.E. Grose, L.A.K. Donev, P.L. McEuen, and D.C. Ralph, Science 306, 86 (2004).

30 B. J. LeRoy, S. G. Lemay, J. Kong, and C. Dekker, Appl. Phys. Lett. 84, 4280 (2004).

${ }^{31}$ L. Y. Gorelik, A. Isacsson, M.V. Voinova, B. Kasemo, R.I. Shekhter, and M. Jonson, Phys. Rev. Lett. 80, 4526 (1998); A. Isacsson, L. Y. Gorelik, M.V. Voinova, B. Kasemo, R.I. Shekhter, and M. Jonson, Physica B 255, 150 (1998).

32 D. Fedorets, L. Y. Gorelik, R.I. Shekhter, and M. Jonson, Phys. Rev. Lett. 92, 166801 (2004).

33 L. M. Jonsson, L. Y. Gorelik, R.I. Shekhter, and M. Jonson, Nano Lett. 5, 1165 (2005).

34 A. Erbe, R. H. Blick, A. Tilke, A. Kriele, and J. P. Kotthaus, Appl. Phys. Lett. 73, 3751 (1998).

35 A. E. Hanna and M. Tinkham, Phys. Rev. B 44, 5919 (1991).

36 A. Zazunov, V. S. Shumeiko, E. N. Bratus', and G. Wendin, Phys. Rev. B 71, 214505 (2005).

37 V. N. Popov, V. E. Van Doren, and M. Balkanski, Phys. Rev. B 61, 3078 (2000). 